\title{
Construction and Functional Analysis of Luciferase Reporter Plasmid Containing CaV1.2 Gene Promoter
}

\author{
Li Man, Gong Huiqin, Zhang jian, Wang Nan, Zhang Tongcun \\ Key Laboratory of Industrial Microbiology \\ Tianjin University of Science and Technology \\ Tianjin, China \\ e-mail:mandylibiology@hotmail.com,goodluck9105@163.com, zhangjian802366@163.com,wn929@tust.edu.cn, \\ tony@tust.edu.cn
}

\begin{abstract}
L-type $\mathrm{Ca}^{2+}$ channels (LTCCs) are the main mediators of $\mathrm{Ca}^{2+}$ influx. LTCCs are multi-subunit complexes, of which CaV1.2 subunit forms the channel pore for $\mathrm{Ca}^{2+}$ movement. $\mathrm{Ca}^{2+}$ influx through LTCC CaV1.2 is a proximal signal for pathological cardiomyocyte hypertrophy. However, very little is known regarding the molecular mechanism of LTCC transcription regulation. Myocardin, a co-activator of serum response factor (SRF) that potently transactivates CArG box-containing cardiac and smooth muscle target genes, can stimulate neonatal rat cardiomyocyte hypertrophy. In the present study, the gene fragment of rat LTCC CaV1.2 promoter containing five CArG boxes or no CArG box was amplified by PCR and cloned into empty vector pGL3-Basic to construct luciferase reporter plasmids, then luciferase activity assay was performed in HEK293T cells. The results showed that the luciferase reporter plasmids were constructed successfully, Myocardin potently activated CArG boxcontaining LTCC CaV1.2 reporter, by contrast, Myocardin had a weaker transcriptional activation on LTCC CaV1.2 reporter containing no CArG box. The results suggest that Myocardin may play a critical role in regulating the expression of LTCC CaV1.2 in cardiomyocyte hypertrophy.
\end{abstract} assay

Keywords-CaV1.2; CArG box; promoter; luciferase activity

\section{INTRODUCTION}

Cardiomyocyte function is predominantly regulated by $\mathrm{Ca}^{2+}$ mobilization in the cytosol and dysregulation of $\mathrm{Ca}^{2+}$ is a central cause of heart disease, including cardiac hypertrophy. L-type $\mathrm{Ca}^{2+}$ channels (LTCCs) are the main mediators of extracellular $\mathrm{Ca}^{2+}$ influx. $\mathrm{Ca}^{2+}$ influx through LTCC regulates excitation-contraction coupling, signal transduction and gene expression. Previous study demonstrated that increasing $\mathrm{Ca}^{2+}$ through LTCC CaV1.2 induced pathological cardiomyocyte hypertrophy by activating calcineurin/nuclear factor of activated $\mathrm{T}$ cells (calcineurin/NFAT) and $\mathrm{Ca}^{2+} /$ calmodulin-dependent protein kinases II/class II histone deacetylases (CaMKII/HDAC) pathways [1]. LTCCs are multi-subunit complexes containing, as a minimum, $\alpha 1 \mathrm{C}$ subunit (CaV1.2), the auxiliary $\beta$ subunit and $\alpha 2 / \delta$ subunit [2]. The $\mathrm{CaV} 1.2$ subunit is the pore-forming protein that responsible for voltage-dependent $\mathrm{Ca}^{2+}$ channel opening and channel selectivity for $\mathrm{Ca}^{2+}$. The majority of studies focus on regulation of CaV1.2 phosphorylation state and channel function [3], very little is known regarding the molecular mechanism of CaV1.2 transcription regulation.

Serum response factor (SRF) is one of the most important transcription factors regulated cardiac hypertrophy. Myocardin, a potent co-activator of SRF, is necessary and sufficient for the expressions of hypertrophic genes by binding the core sequence of $\mathrm{CArG}$ box (CC $\left.[\mathrm{A} / \mathrm{T}]_{6} \mathrm{GG}\right)$ as a homodimer [4]. We have previously shown that overexpression of Myocardin can stimulate neonatal rat cardiomyocyte hypertrophy [5]. Recent studies have shown that $\mathrm{Ca}^{2+}$ influx induces the activation of calcineurin, which leads to the translocation of NFAT and the transcription of Myocardin [6, 7]. However, the exact mechanism of Myocardin-induced cardiomyocyte hypertrophy is not clear. Bioinformatics analysis showed that LTCC CaV1.2 promoter contained several CArG boxes, suggesting that Myocardin may transactivate CaV1.2 gene expression.

In this study, the gene fragment of rat LTCC CaV1.2 promoter containing five CArG boxes or no CArG box was amplified by PCR and cloned into empty vector pGL3Basic to construct luciferase reporter plasmids, then luciferase activity assay was performed in HEK293T cells. The results showed that the luciferase reporter plasmids were constructed successfully and Myocardin could potently activate $\mathrm{CArG}$ box-containing $\mathrm{CaV} 1.2$ reporter, by contrast, Myocardin had a weaker transcriptional activation on CaV1.2 reporter containing no CArG box. The results suggest that Myocardin may play a critical role in regulating the expression of LTCC CaV1.2 in cardiomyocyte hypertrophy.

\section{Materials And Methods}

\section{A. Cell Culture and Transfection}

HEK293T cell was obtained from American Type Culture Collection (ATCC) and it was cultured with high glucose Dulbecco's modified Eagle's medium (DMEM) (GIBCO) supplemented with 10\% fetal bovine serum (FBS) at $37^{\circ} \mathrm{C}$ in a $5 \% \mathrm{CO} 2$ incubator.

For transfection experiments, HEK293T cells were cultured in DMEM medium without antibiotics when they were $50-60 \%$ confluent. Transfection was carried out with 
transfection reagent (Lipofectamine 2000 Reagent; Invitrogen)

\section{B. Plasmid Construction}

The DNA fragments of CaV1.2 promoter (CaV1.23235-Luc, -3193 to +42 ; CaV1.2-528-Luc, -486 to +42 ) were amplified by PCR and then cloned into pGL3 luciferase reporter vectors, respectively. The primers used in PCR reactions were as follows: $\mathrm{CaV} 1.2-3235-\mathrm{Luc}$ : forward primer-ATTGGTACCATTCGGTGTTTGAGGTATCTGG, reverse CGAAAAGCTTAATCATTGTGACTCCAGTTGG; CaV1.2-528-Luc: forward primerGGCGGTACCTCCTTAAAATAGTTCTGCAGCT, reverse primer-

CGAAAAGCTTAATCATTGTGACTCCAGTTGG.Amplif ication conditions for PCR are as follows: CaV1.2-3235Luc: pre-degeneration for $5 \mathrm{~min}$ at $94^{\circ} \mathrm{C}$, denaturation for $45 \mathrm{~s}$ at $94^{\circ} \mathrm{C}$, annealing for $30 \mathrm{~s}$ at $58^{\circ} \mathrm{C}$ and extension for 3 min $30 \mathrm{~s}$ at $72^{\circ} \mathrm{C}$. CaV1.2-528-Luc: pre-degeneration for 5 min at $94^{\circ} \mathrm{C}$, denaturation for $45 \mathrm{~s}$ at $94^{\circ} \mathrm{C}$, annealing for $30 \mathrm{~s}$ at $54^{\circ} \mathrm{C}$ and extension for $1 \mathrm{~min}$ at $72^{\circ} \mathrm{C}$. The $\mathrm{PCR}$ reaction was carried out for 35 cycles.

The gene fragments of rat LTCC CaV1.2 promoter and pGL3-Basic vector were digested with restriction enzyme $K p n I$ and HindIII at $37^{\circ} \mathrm{C}$ for $1 \mathrm{~h}$ and then the gene fragments were ligated to vector pGL3-Basic using T4 DNA ligase at $16^{\circ} \mathrm{C}$ for $24 \mathrm{~h}$. The recombinant plasmids were transformed into E. coli DH5 $\alpha$ and confirmed by restriction enzyme digestion and DNA sequencing.

\section{Luciferase Reporter Assays}

Luciferase activity assay was performed using the Luciferase Assay System (Promega) according to the instructions. Briefly, the transfected cells were lysed in Cell Culture Lysis Reagent. $20 \mu \mathrm{L}$ of cell lysate was added into a 96-well enzyme label plate and reading was initiated by the injection of $100 \quad \mu \mathrm{L}$ of Luciferase Assay Reagent into the plate on a Synergy ${ }^{\text {TM }} 4$ (Bioteck).

\section{Statistical analyses}

All experiments were performed three times. Data were analyzed with unpaired student $\mathrm{t}$ test. Two tailed $P$ value $<0.01$ was indicated by $* *$. Data were presented as Mean \pm SD.

\section{RESULTS}

\section{A. Construction of LTCC CaV1.2 Luciferase Reporter Plasmids}

A structure schematic of CArG box-containing LTCC CaV1.2 reporter plasmid (CaV1.2-3235-Luc) and truncated CaV1.2 reporter plasmid (CaV1.2-528-Luc) are shown in Figure 1. CaV1.2-3235-Luc contains 5 CArG boxes in the promoter region while $\mathrm{CaV} 1.2-528$-Luc contains no $\mathrm{CArG}$ box and they were $3235 \mathrm{bp}$ and $528 \mathrm{bp}$ respectively. $1 \%$ agarose gel electrophoresis was used to analyze the PCR amplification products. As shown in Figure 2A, a 3235bp DNA fragment was amplified specifically by PCR and as shown in Figure 2B, of these PCR products, a 528bp DNA fragment can be significantly distinguished.

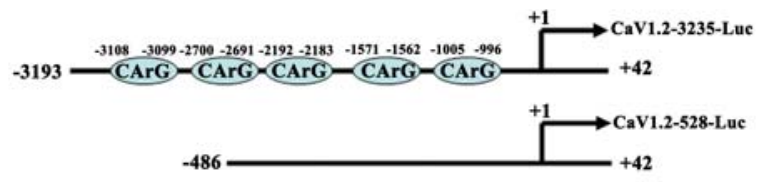

Figure 1. A structure schematic of CaV1.2 Luciferase Reporter Plasmids.
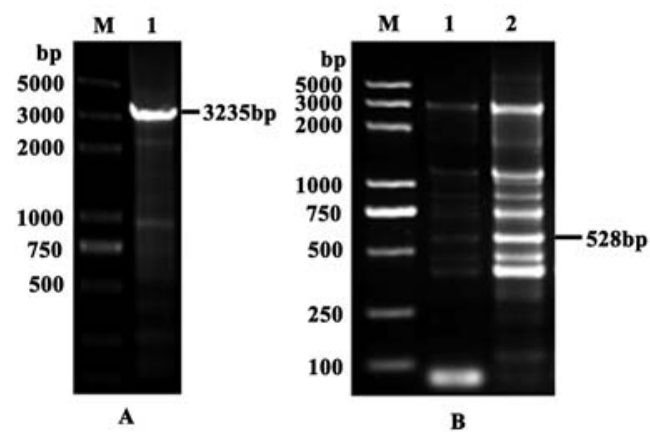

Figure 2. Agarose gel electrophoretic analysis of PCR amplification products.

A. PCR products of CArG box-containing CaV1.2 promoter (M: DL2000 Plus Marker;1: PCR products) B. PCR products of truncated CaV1.2 promoter (M: DL2000 Plus Marker; 1-2: PCR products)

After restriction enzyme digestion and recombinant, the recombinant plasmids were extracted and purified, and the $1 \%$ agarose gel electrophoretic analysis was performed. As shown in Figure $3 \mathrm{~A}$ and $3 \mathrm{~B}$, the size of recombinant plasmids were larger than vector pGL3-Basic.

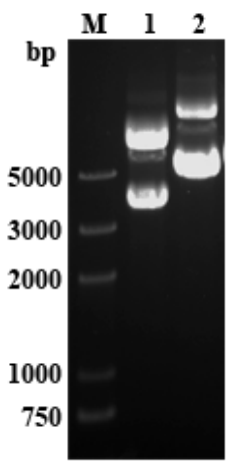

A

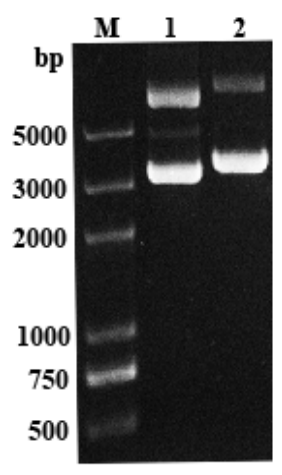

B
Figure 3. Agarose gel electrophoretic analysis of recombinant plasmids. A. Electropherogram of CaV1.2-3235-Luc recombinant plasmids (M: DL2000 Plus Marker,1: pGL3-Basic vector; 2: CaV1.2-3235-Luc recombinant plasmids) B. Electropherogram of CaV1.2-528-Luc recombinant plasmids(M: DL2000 Plus Marker,1: pGL3-Basic vector; 2: CaV1.2-528-Luc recombinant plasmids)

Next, the recombinant plasmids were digested with restriction enzyme KpnI and HindIII at $37^{\circ} \mathrm{C}$ for $1 \mathrm{~h}$ and then the restricted DNA products were separated by $2 \%$ agarose gel electrophoresisand. As shown in Figure 4A, the sepharose electrophoresis of DNA showed 3235bp and 4818 bp two bands, suggesting that CaV1.2-3235-Luc was successfully constructed. Figure 4B showed that the 
recombinant plasmid was digested into a $528 \mathrm{bp}$ fragment and a 4818bp fragment, suggesting that CaV1.2-528-Luc was constructed successfully.
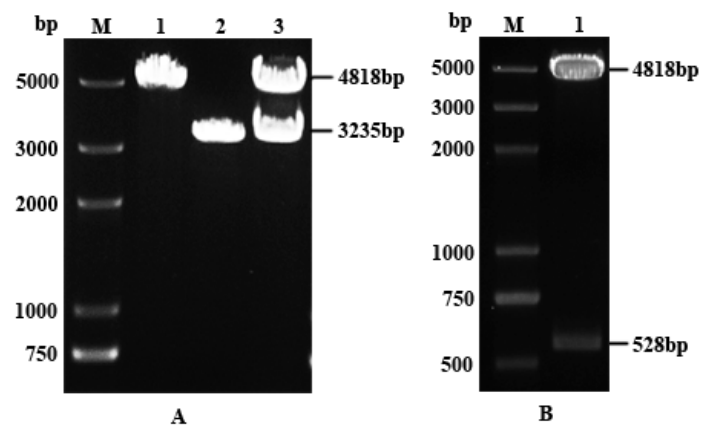

Figure 4. Restriction enzyme digestion analysis of recombinant plasmids A. Restriction enzyme digestion identification of CaV1.2-3235-Luc (M: DL2000 Plus Marker,1: Double digestion of pGL3-Basic vector; 2: Double digestion of 3235bp fragment; 3: Double digestion of CaV1.2-3235-Luc)

B. Restriction enzyme digestion identification of CaV1.2-528-Luc (M: DL2000 Plus Marker; 1: Double digestion of CaV1.2-528-Luc)

Finally, by sequence analysis, the recombinant plasmids of CaV1.2-3235-Luc and CaV1.2-528-Luc were successfully constructed.

\section{B. Luciferase Assay}

Next, we analyzed whether CaV1.2 transcriptional activity might be affected by overexpression of Myocardin. The CaV1.2-3235-Luc plasmids or CaV1.2-528-Luc plasmids were co-transfected with pcDNA3.1-Myocardin expression plasmids or pcDNA3.1 vectors using Lipofectamine 2000 for $24 \mathrm{~h}$. As shown in Figure 5, the activity of the CaV1.2-3235-Luc was significantly activated by overexpression of Myocardin, approximately 14-fold, while Myocardin increased CaV1.2528-Luc activity by approximately 6-fold, which had significant difference compared with control group. However, the activation of CaV1.2-528-Luc induced by Myocardin was strongly suppressed compared with the activation of CaV1.2-3235-Luc induced by Myocardin, suggesting that Myocardin transactivated the expression of CaV1.2 probably via a CArG-dependent mechanism.

\section{Discussion}

Cardiac hypertrophy is the common compensatory response to a number of physiological and pathological stimulus. There is growing evidence for a pathophysiological role of $\mathrm{Ca}^{2+}$ overload in cardiac hypertrophy [8]. LTCC is the membrane ion channel that most responsible for $\mathrm{Ca}^{2+}$ influx and excitation-contraction coupling. Cav1.2 is the pore-forming subunit of LTCC. $\mathrm{Ca}^{2+}$ influx through LTCC CaV1.2 is sufficient to induce pathological cardiac hypertrophy by activating calcineurin/NFAT and CaMKII/HDAC pathways [1]. Several studies over the years focus on regulation of CaV1.2 phosphorylation state and channel function. CaV1.2 channel is regulated by different kinases, including cGMPdependent protein kinase, cAMP-dependent protein kinase, and protein kinase $\mathrm{C}[9]$.

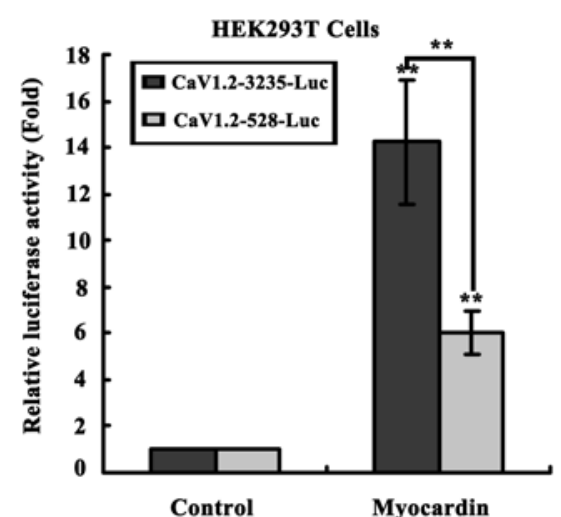

Figure 5. Myocardin increases the transcriptional activity of LTCC CaV1.2 promoter.

However, very little is known regarding the molecular mechanism of CaV1.2 transcription regulation in cardiac hypertrophy. It has been shown that nuclear factor-kappaB (NFKB) stimulates functional CaV1.2 expression in cerebral artery myocytes [10]. Endothelin-1 stimulation increases the LTCC CaV1.2 transcription in cardiomyocytes via activation of extracellular signal-regulated kinase $1 / 2$ pathway [11]. Our present study demonstrated that Myocardin regulated transcriptional activity of LTCC CaV1.2 via binding to CArG boxes.

Myocardin has been demonstrated to play an important role in $\mathrm{Ca}^{2+}$ signal-induced cardiomyocyte hypertrophy [6]. However, the molecular mechanism of this response is not unclear. These results preliminarily demonstrated that Myocardin regulating CaV1.2 expression may contribute to Myocardin-induced cardiomyocyte hypertrophy. However, further study is needed to understand the exact mechanism of Myocardin-induced CaV1.2 expression.

\section{ACKNOWLEDGMENTS}

This work was financially supported by National Natural Science Foundation of China (No. 31171303, 31070694, 31270837), Program for Changjiang Scholars and Innovative Research Team in University of Ministry of Education of China (IRT1166), Research Fund for the Doctoral Program of Higher Education of China (20111208110001) and Natural Science Foundation of Tianjin University of Science \& Technology (20130109).

\section{REFERENCES}

[1] X.W. Chen H. Nakayama, X. Zhang, X. Ai, D .M. Harris et al., "Calcium influx through Cav1.2 is a proximal signal for pathological cardiomyocyte hypertrophy," J Mol Cell Cardiol, vol. 50, Mar. 2011, pp 460-470, doi: 10.1016/j.yjmcc.

[2] R.M. Shaw, and H.M. Colecraft, "L-type calcium channel targeting and local signalling in cardiac myocytes," Cardiovasc Res, vol. 98, May 2013, pp 177-186, doi: 10.1093/cvr/cvt021.

[3] S. Tandan_ , Y. Wang, T.T. Wang N . Jiang, D.D. Hall, et al., "Physical and functional interaction between calcineurin and the cardiac L-type Ca2+ channel," Circ Res, vol. 105, Jul. 2009, pp 51-60, doi: 10.1161/CIRCRESAHA.109.199828. 
[4] N. Frey and E.N. Olson "Cardiac hypertrophy: the good, the bad, and the ugly," Annu Rev Physiol, vol. 65, 2003, pp 45-79.

[5] W. Xing, T.C. Zhang D. Cag Z. Wang CL. Antos, et al., "Myocardin induces cardiomyocyte hypertrophy," Circ Res vol. 98, Apr. 2006, pp 1089-1097.

[6] M. Li, N. Wang, H.Q. Gong, W.Z. Li, X.H. Liao, et al., "Ca2+ signalinduced cardiomyocyte hypertrophy through activation of myocardin," Gene, vol. 557, Feb. 2015, pp 43-51, doi: 10.1016/j.gene.2014.12.007.

[7] K. Wang, B. Long, J. Zhou and P.F. Li, "miR-9 and NFATc3 regulate myocardin in cardiac hypertrophy," J Bio Chem, vol. 285, Apr. 2010, pp 11903-11912, doi:10.1074/jbc.M109.098004.

[8] S.R. Houses and J.D. Molkentin, "Does Contractile Ca2+ Control Calcineurin-NFAT Signaling and Pathological Hypertrophy in Cardiac
Myocytes?" Sci Signal, vol. 1, Jun. 2008, pp pe31, doi: 10.1126/scisignal.125pe31.

[9] K.D. Keef, J.R. Hume and J. Zhong, "Regulation of cardiac and smooth muscle Ca2+ channels (Cav1.2a,b) by protein kinases," Am J Physiol Cell Physiol, vol. 281, Dec. 2001, pp 1743-1756.

[10]D. Narayannan, Q. Xi, L.M. Pfeffer and J.H. Jaqqar, "Mitochondria Control Functional CaV1.2 Expression in Smooth Muscle Cells of Cerebral Arteries," Circ Res, vol.107, Sep. 2010, pp 631-641, doi: 10.1161/CIRCRESAHA. 110.224345

[11] L. Yu, M. Li, T. She, C. Shi, W. Meng, et al.,"Endothelin-1 stimulates the expression of L-type $\mathrm{Ca} 2+$ channels in neonatal rat cardiomyocytes via the extracellular signal-regulated kinase $1 / 2$ pathway," J Membr Biol, vol. 246, Apr. 2013, pp 343-353, doi: 10.1007/s00232-0139538-7. 